\title{
OPTIMAL CONTROL FOR A NONLINEAR SYSTEM DESCRIBING THE MECHANOCHEMICAL PROCESS IN CELL MORPHOGENESIS
}

\author{
LAHCEN GHANNAM ${ }^{1}$
}

\begin{abstract}
In this paper a system of partial differential equations is considered that constitutes a one-dimensional mechanochemical model of cellular morphogenesis, based on calcium ion regulation of the viscoelastic properties of cellular cortex. Calcium input and out put processes, as well as diffusive effects are accounted in the description of the cell dynamics. The stability behavior under distributed loads and calcium sources at the boundary is investigated. An optimal control problem is formulated which uses loads and calcium sources as control variables. The existence of optimal controls is proved.

Résumé. Cet article est consacré à l'étude du contrôle optimal d'un système parabolique non linéaire qui modèlise la morphogenèse cellulaire. Ce modèle est basé sur l'interaction entre le calcium et les propriétés viscoélastiques du cytoplasme cellulaire. On montre un résultat de continuité par rapport aux données du problème ainsi que l'existence d'un contrôle, sans toutefois expliciter les conditions d'optimalité.
\end{abstract}

\section{INTRODUCTION}

In this paper, we consider the following nonlinear partial differential equations

$$
\begin{gathered}
u_{t t}=\left(\frac{\partial \Psi}{\partial \varepsilon}(\varepsilon, \chi)+\mu_{1}(\chi) u_{x t}\right)_{x}-E(\chi) u+f(x, t) \quad \text { in } \Omega_{T} \\
\chi_{t}=D \chi_{x x}+(K-\chi) \phi(\varepsilon)-\mu_{2}(\chi) \quad \text { in } \Omega_{T} \\
\varepsilon=u_{x},
\end{gathered}
$$

together with the initial data

$$
u(., 0)=u_{0}, \quad u_{t}(., 0)=u_{1} \text { et } \quad \chi(., 0)=\chi_{0} \quad \text { in } \quad \Omega
$$

and the boundary conditions

$$
\begin{gathered}
u(0, t)=0,\left(\frac{\partial \Psi}{\partial \varepsilon}(\varepsilon, \chi)+\mu_{1}(\chi) u_{x t}\right)(1, t)=P(t) \quad \text { on } \quad \Gamma_{T} \\
-D \chi_{\nu}^{\prime}=k\left(\chi-\chi_{\Gamma}\right) \quad \text { on } \quad \Gamma_{T}
\end{gathered}
$$

where $\Omega=(0,1) \subset \mathbb{R}, \Omega_{T}=\Omega \times(0, T), \quad \Gamma=\{0,1\}, \quad \Gamma_{T}=\Gamma \times(0, T)$ and $\chi_{\nu}^{\prime}=-\chi_{x}$ at $x=0, \quad \chi_{\nu}^{\prime}=\chi_{x}$ at $x=1$.

\footnotetext{
1 Laboratoire MIP-UMR 5640, Université Paul Sabatier, UFR MIG, 118, route de Narbonne, F-31062 Toulouse Cedex 04, FRANCE; e-mail: ghannam@cict.fr 
Equations (1)-(6) constitute a one-dimensional model to describe the pattern evolution for a cell growing under the influence of calcium (compare $[3,7,12] \ldots$, for a detailed description of the physical background and the modeling). In this connection, the physical meaning of the involved quantities are

The unknowns $u$ and $\chi$ represent respectively the displacement and the free calcium (local) concentration at a point $x$ of the medium (cytogel) $\Omega . \Psi$ is the free energy, $f$ the distributed loads, $\varepsilon=u_{x}$ the strain, $\frac{\partial \Psi}{\partial \varepsilon}$ the stress, $u_{x t}$ the viscous (dissipative) stress, $E(\chi)$ a positive factor of the restoring forces, $\chi_{\Gamma}$ a calcium concentration in the surrounding medium. $D, K$ and $k$ are positive constants representing respectively the diffusion coefficient, the total calcium concentration(binding and release). $\Phi\left(u_{x}\right)$ is a rate which takes account of stretching or compression effects on calcium release or calcium binding. The local mass density $\rho$ of the material is assumed to be constant and normalized to unity. A typical form of $\mu_{2}$ is $\mu_{2}(\chi)=|\chi|^{n-1} \chi$ with $n \geq 1$, where the integer $n$ is the stoïchiometry of the reaction ( $\mathrm{cf}[7])$. Here, we note also that the free energy $\Psi(\varepsilon, \chi)$ is not convex with respect to $\varepsilon$.

Previous papers concerned with (1)-(6) deal with the questions of modeling, existence and uniqueness. The aim of this paper is twofold: Firstly, we establish a stability result of the solution with respect to the data $f$, $P, \chi_{\Gamma}$; secondly, we are concerned with the optimal control of the evolution of cytogel (which is considered as the medium) using the data $f, P$ and $\chi$ as control variables. We shall formulate the control problem and prove the existence of optimal controls.

\section{Existence AND UNIQUENESS RESUltS}

For the sequel we impose the following structural hypotheses on the model.

(H1) $\Psi \in \mathcal{C}^{3}\left(\mathbb{R}^{2}\right)$ has the following properties:

$$
\left\{\begin{array}{l}
\frac{\partial \Psi}{\partial \varepsilon}(\varepsilon, \chi)\left|\leq c_{1}\right| \varepsilon \mid \\
\frac{\partial^{2} \Psi}{\partial \varepsilon \partial \chi}(\varepsilon, \chi)\left|\leq c_{2}\right| \varepsilon \mid, \\
\frac{\partial^{2} \Psi}{\partial \varepsilon^{2}}(\varepsilon, \chi) \mid \leq c_{3} \text { for all } \chi \in \mathbb{R} \text { and }|\varepsilon| \geq \varepsilon_{0} \text { for some } \varepsilon_{0}>0,
\end{array}\right.
$$

(H2) $E$ in $\mathcal{C}^{1}(\mathbb{R})$ and $|E(\chi)| \leq c_{4}$ for all $\chi \in \mathbb{R}$,

(H3) $\mu_{1}$ in $\mathcal{C}^{1}(\mathbb{R}), \mu_{1}(\chi) \geq c_{5}>0$ for all $\chi \in \mathbb{R}$,

(H4) $\mu_{2}$ in $\mathcal{C}^{1}(\mathbb{R})$ and $\mu_{2}$ is increasing and $\mu_{2}(0)=0$,

(H5) $\phi$ in $\mathcal{C}^{1}(\mathbb{R})$ and $|\phi(\varepsilon)| \leq c_{6}|\varepsilon|+c_{7}$ for all $\varepsilon \in \mathbb{R}$,

with positive constants $c_{i}, i=1, \ldots, 6$.

We introduce the following Hilbert space

$$
V=\left\{v \in H^{1}(\Omega) / v(0)=0\right\}
$$

endowed with the norm $\|v\|_{V}=\left(\int_{\Omega}\left|v_{x}\right|^{2}\right)^{\frac{1}{2}}$.

Henceforth we shall denote by $\langle$,$\rangle either the scalar product in L^{2}(\Omega)$ or the duality pairing between $V^{\prime}$ and $V$ or between $\left(H^{1}(\Omega)\right)^{\prime}$ and $H^{1}(\Omega)$. Also, for the sake of simplicity, the notation $\|\cdot\|$ will be used to indicate the norm in $L^{2}(\Omega)$.

In addition to the structural hypothesis (H1)-(H4), we assume the following for the data of the problem :

(H6) $u_{0} \in V \cap H^{2}(\Omega), u_{1} \in V$,

(H7) $\chi_{0} \in H^{2}(\Omega)$,

(H8) $f \in L^{2}\left(\Omega_{T}\right)$,

(H9) $P \in L^{2}(0, T)$,

(H10) $\chi_{\Gamma} \in\left(H^{1}(0, T)\right)^{2}$,

and the compatibility condition: $-D\left(\chi_{0}\right)_{\nu}^{\prime}=k\left(\chi_{0}-\chi_{\Gamma}\right) \quad$ on $\quad \Gamma$. 
Here follows the variational formulation of the problem (1)-(6).

Problem $(\boldsymbol{P})$. Find a pair $(u, \chi)$ of functions defined on $(0, T)$ such that:

$$
\begin{aligned}
& u \in H^{2}\left(0, T, L^{2}(\Omega)\right) \cap H^{1}\left(0, T, V \cap H^{2}(\Omega)\right) . \\
& \chi \in H^{1}\left(0, T, H^{1}(\Omega)\right) \cap L^{2}\left(0, T, H^{2}(\Omega)\right) . \\
& \left\langle u_{t t}, v\right\rangle+\left\langle\frac{\partial \Psi}{\partial \varepsilon}\left(u_{x}, \chi\right)+\mu_{1}(\chi) u_{x t}, v_{x}\right\rangle=\langle-E(\chi) u+f, v\rangle+P(t) v(1) \\
& \forall v \in V \text {; a.e in }(0, T) \\
& \left\langle\chi_{t}, \theta\right\rangle+D\left\langle\chi_{x}, \theta_{x}\right\rangle+\left\langle\mu_{2}(\chi), \theta\right\rangle+k \int_{\Gamma}\left(\chi-\chi_{\Gamma}\right) \theta d x=\left\langle(K-\chi) \phi\left(u_{x}\right), \theta\right\rangle \\
& u(., 0)=u_{0}, \quad u_{t}(., 0)=u_{1} \text {, et } \quad \chi(., 0)=\chi_{0} \quad \text { a.e in } \quad \Omega .
\end{aligned}
$$

The solution of Problem $(P)$ is said weak solution of the system (1)-(6).

Theorem 2.1. Under hypotheses (H1)-(H10), the Problem (P) admits a unique global solution (u, $\chi)$ such that: $u_{x t} \in L^{\infty}\left(0, T, L^{2}(\Omega)\right)$.

$\chi \in L^{\infty}\left(0, T, H^{2}(\Omega)\right)$.

Proof. The result follows by rather obvious modifications of the proof of Theorem 3.1 in [6] .

\section{The Control PROBlem}

\subsection{Continuity of the solution operator}

Suppose that the general hypotheses (H1)-(H10) are satisfied. According to the Theorem 2.1, we know that to any $\left(f, P, \chi_{\Gamma}\right) \in L^{2}\left(\Omega_{T}\right) \times L^{2}(0, T) \times\left(H^{1}(0, T)\right)^{2}$, the system (1)-(6) admits a unique weak solution

$$
(u, \chi) \in\left(H^{2}\left(0, T, L^{2}(\Omega)\right) \cap H^{1}\left(0, T, V \cap H^{2}(\Omega)\right)\right) \times\left(H^{1}\left(0, T, H^{1}(\Omega)\right) \cap L^{\infty}\left(0, T, H^{2}(\Omega)\right)\right) .
$$

Our aim is to investigate the continuity properties of the mapping $\left(f, P, \chi_{\Gamma}\right) \rightarrow(u, \chi)$.

Let $B_{R}$ denote the nonempty closed and convex set

$$
B_{R}=\left\{(f, P, \vartheta) \in L^{2}\left(\Omega_{T}\right) \times L^{2}(0, T) \times\left(H^{1}(0, T)\right)^{2} /\|f\|_{L^{2}\left(\Omega_{T}\right)}+\|P\|_{L^{2}(0, T)}+\|\vartheta\|_{\left(H^{1}(0, T)\right)^{2}} \leq R\right\}
$$

Now let $\left(f^{(i)}, P^{(i)}, \chi_{\Gamma}^{(i)}\right) \in B_{R}$ and let $\left(u^{(i)}, \chi^{(i)}\right)(i=1,2)$ denote the corresponding weak solutions of (1)-(6). There holds:

Theorem 3.1. Suppose the assumptions of Theorem 2.1 are true, then the solution operator $\left(f, P, \chi_{\Gamma}\right) \rightarrow(u, \chi)$ is locally Lipschitz continuous in the following sense:

To any $R>0$, there exists a constant $c$ such that: $\forall\left(f^{(i)}, P^{(i)}, \chi_{\Gamma}^{(i)}\right) \in B_{R}(i=1,2)$,

$$
\begin{aligned}
\sup _{t \in[0, T]} & \left(\left\|\chi_{x}^{(1)}-\chi_{x}^{(2)}\right\|_{L^{2}(\Omega)}^{2}+\left\|\chi^{(1)}-\chi^{(2)}\right\|_{L^{2}(\Gamma)}^{2}+\left\|u_{t}^{(1)}-u_{t}^{(2)}\right\|_{L^{2}(\Omega)}^{2}+\right. \\
+ & \left.\left\|u_{x}^{(1)}-u_{x}^{(2)}\right\|_{L^{2}(\Omega)}^{2}\right)+\left\|\chi_{t}^{(1)}-\chi_{t}^{(2)}\right\|_{L^{2}\left(\Omega_{T}\right)}^{2}+\left\|u_{x t}^{(1)}-u_{x t}^{(2)}\right\|_{L^{2}\left(\Omega_{T}\right)}^{2} \\
& \leq c\left(\left\|f^{(1)}-f^{(2)}\right\|_{L^{2}\left(\Omega_{T}\right)}^{2}+\left\|P^{(1)}-P^{(2)}\right\|_{L^{2}(0, T)}^{2}+\left\|\chi_{\Gamma}^{(1)}-\chi_{\Gamma}^{(2)}\right\|_{\left(H^{1}(0, T)\right)^{2}}^{2}\right)
\end{aligned}
$$


Proof. We put $w=u^{(1)}-u^{(2)}$ and $\xi=\chi^{(1)}-\chi^{(2)}$. Then from (7) and (8) we deduce that:

$$
\begin{aligned}
\int_{\Omega} w_{t t} v d x+\left(\mu_{1}\left(\chi^{(1)}\right) w_{x t}+w_{x}\right) v_{x} d x=- & \int_{\Omega}\left(\frac{\partial \Psi}{\partial \varepsilon}\left(u_{x}^{(1)}, \chi^{(1)}\right)-\frac{\partial \Psi}{\partial \varepsilon}\left(u_{x}^{(2)}, \chi^{(1)}\right)\right) v_{x} d x+ \\
& +\int_{\Omega}\left(\mu_{1}\left(\chi^{(2)}\right)-\mu_{1}\left(\chi^{(1)}\right)\right) u_{x t}^{(2)} v_{x}- \\
& -\int_{\Omega}\left(\frac{\partial \Psi}{\partial \varepsilon}\left(u_{x}^{(2)}, \chi^{(1)}\right)-\frac{\partial \Psi}{\partial \varepsilon}\left(u_{x}^{(2)}, \chi^{(2)}\right)-w_{x}\right) v_{x} d x+ \\
& +\int_{\Omega}\left(-E\left(\chi^{(1)}\right) w-\left(E\left(\chi^{(1)}\right)-E\left(\chi^{(2)}\right)\right) u^{(2)}+f^{(1)}-f^{(2)}\right) v d x+ \\
& +\left(P^{(1)}(t)-P^{(2)}(t)\right) v(1)
\end{aligned}
$$

$$
\begin{aligned}
\int_{\Omega}\left(\xi_{t} \theta+D \xi_{x} \theta_{x}+\left(\mu_{2}\left(\chi^{(1)}\right)-\mu_{2}\left(\chi^{(2)}\right)\right) \theta\right) d x+k \int_{\Gamma} \xi \theta d x= & k \int_{\Gamma}\left(\chi_{\Gamma}^{(1)}-\chi_{\Gamma}^{(2)}\right) \theta d x-\int_{\Omega} \xi \phi\left(u_{x}^{(1)}\right) \theta d x+ \\
& +\int_{\Omega}\left(K-\chi^{(2)}\right)\left(\phi\left(u_{x}^{(1)}\right)-\phi\left(u_{x}^{(2)}\right)\right) \theta d x
\end{aligned}
$$

Recall that $w_{t}(0, t)=0$ in view of boundary condition (5).

Inserting $v=w_{t}$ in (9) gives upon integration over $(0, t)$ :

$$
\begin{aligned}
\frac{1}{2}\left(\left\|w_{x}(t)\right\|^{2}+\left\|w_{t}(t)\right\|^{2}\right)+\int_{0}^{t} \int_{\Omega} \mu_{1}\left(\chi_{1}\right) w_{x t}^{2}(x, s) d x d s= & \int_{0}^{t}\left(P^{(1)}(t)-P^{(2)}(t)\right) w_{t}(1, t)- \\
& -\int_{0}^{t} \int_{\Omega}\left(\frac{\partial \Psi}{\partial \varepsilon}\left(u_{x}^{(1)}, \chi^{(1)}\right)-\frac{\partial \Psi}{\partial \varepsilon}\left(u_{x}^{(2)}, \chi^{(1)}\right)\right) w_{x t}+ \\
& +\int_{0}^{t} \int_{\Omega} w_{x} w_{x t}+\int_{0}^{t} \int_{\Omega}\left(\mu_{1}\left(\chi^{(1)}\right)-\mu_{1}\left(\chi^{(2)}\right)\right) u_{x t}^{(2)} w_{x t}+ \\
& +\int_{0}^{t} \int_{\Omega}\left(\frac{\partial \Psi}{\partial \varepsilon}\left(u_{x}^{(2)}, \chi^{(1)}\right)-\frac{\partial \Psi}{\partial \varepsilon}\left(u_{x}^{(2)}, \chi^{(2)}\right)\right) w_{x t}- \\
& -\int_{0}^{t} \int_{\Omega} E\left(\chi^{(1)}\right) w w_{t}+\int_{0}^{t} \int\left(f^{(1)}-f^{(2)}\right) w_{t}- \\
& -\int_{0}^{t} \int_{\Omega}\left(E\left(\chi^{(1)}\right)-E\left(\chi^{(2)}\right)\right) u^{(2)} w_{t} .
\end{aligned}
$$

Consider the individual terms in (11).

By Young's inequality

$$
\int_{0}^{t} \int_{\Omega} w_{x} w_{x t} \leq \int_{0}^{t}\left(\frac{1}{2 \delta}\left\|w_{x}\right\|^{2}+\frac{\delta}{2}\left\|w_{x t}\right\|^{2}\right) d s
$$


Now let $(x, \tau) \in \Omega_{T}$ be arbitrary. By Taylor's formula, we have

$$
\frac{\partial \Psi}{\partial \varepsilon}\left(u_{x}^{(1)}(x, \tau), \chi^{(1)}(x, \tau)\right)-\frac{\partial \Psi}{\partial \varepsilon}\left(u_{x}^{(2)}(x, \tau), \chi^{(1)}(x, \tau)\right)=\frac{\partial^{2} \Psi}{\partial \varepsilon^{2}}(\lambda, \vartheta) w_{x}(x, \tau)+\frac{\partial^{2} \Psi}{\partial \varepsilon \partial \chi}(\lambda, \vartheta) \xi(x, \tau)
$$

where $(\lambda, \vartheta)$ lies in the segment between the points $\left(u_{x}^{(1)}(x, \tau), \chi^{(1)}(x, \tau)\right)$ and $\left(u_{x}^{(2)}(x, \tau), \chi^{(2)}(x, \tau)\right)$. Thus, using (H1) and the fact that $u_{x}^{(i)}$ and $\chi^{(i)}(i=1,2)$ are bounded in $L^{\infty}\left(\Omega_{T}\right)$ (cf $\left.[6]\right)$, we obtain:

$$
\left|\frac{\partial \Psi}{\partial \varepsilon}\left(u_{x}^{(1)}(x, \tau), \chi^{(1)}(x, \tau)\right)-\frac{\partial \Psi}{\partial \varepsilon}\left(u_{x}^{(2)}(x, \tau), \chi^{(2)}(x, \tau)\right)\right| \leq c\left(\left|w_{x}(x, \tau)\right|+|\xi(x, \tau)|\right)
$$

Hence we can conclude from Young's inequality that with some constant $c>0$, which only depends on $R$,

$$
\begin{aligned}
& \mid \int_{0}^{t} \int_{\Omega}\left(\frac{\partial \Psi}{\partial \varepsilon}\left(u_{x}^{(1)}, \chi^{(1)}\right)\right.\left.-\frac{\partial \Psi}{\partial \varepsilon}\left(u_{x}^{(2)}, \chi^{(1)}\right)\right) w_{x t} \mid+ \\
&+\left|\int_{0}^{t} \int_{\Omega} w_{x} w_{x t}\right| \leq \int_{0}^{t}\left(\frac{c}{2 \delta}\left\|w_{x}\right\|^{2}+\frac{\delta}{2}\left\|w_{x t}\right\|^{2}\right) d s
\end{aligned}
$$

and

$$
\begin{aligned}
\mid \int_{0}^{t} \int_{\Omega}\left(\frac{\partial \Psi}{\partial \varepsilon}\left(u_{x}^{(2)}, \chi^{(1)}\right)\right. & \left.-\frac{\partial \Psi}{\partial \varepsilon}\left(u_{x}^{(2)}, \chi^{(2)}\right)\right) w_{x t} \mid \\
& \leq \int_{0}^{t}\left(\frac{c}{2 \delta}\|\xi\|^{2}+\frac{\delta}{2}\left\|w_{x t}\right\|^{2}\right) d s
\end{aligned}
$$

Now we observe that Theorem 2.1 implies for $\mathrm{i}=1,2$ :

$$
\sup _{s \in(0, T)}\left\|u_{x t}^{(2)}(s)\right\| \leq C
$$

Moreover, for any $(x, s) \in \Omega_{t}$,

$$
|\xi(x, s)| \leq|\xi(0, s)|+\left\|\xi_{x}(s .)\right\|
$$

Due to hypothesis (H3), by mean value Theorem and Young's inequality,

$$
\begin{aligned}
& \mid \int_{0}^{t} \int_{\Omega}\left(\mu_{1}\left(\chi^{(1)}\right)\right.\left.-\mu_{1}\left(\chi^{(2)}\right)\right) u_{x t}^{(2)} w_{x t} \mid \\
& \leq c \int_{0}^{t}\|\xi\|_{\infty}\left\|u_{x t}^{(2)}\right\|\left\|w_{x t}\right\| \\
& \leq \sup _{t \in(0, T)}\left\|u_{x t}^{(2)}(t)\right\|^{2} \int_{0}^{t} \frac{c}{2 \delta}\left(\|\xi\|_{L^{2}(\Gamma)}^{2}+\left\|\xi_{x}\right\|^{2}\right)+\frac{\delta}{2} \int_{0}^{t}\left\|w_{x t}\right\|^{2}
\end{aligned}
$$

and

$$
\int_{0}^{t} \int_{0}^{1}\left(E\left(\chi^{(1)}\right)-E\left(\chi^{(2)}\right)\right) u_{x t}^{(2)} w_{t} \leq \int_{0}^{t}\left(\frac{c}{2}\|\xi\|^{2}+\frac{1}{2}\left\|w_{t}\right\|^{2}\right)
$$


Moreover, we have :

$$
\begin{aligned}
\int_{0}^{t}\left(P^{(1)}(t)-P^{(2)}(t)\right) w_{t}(1, t) & \\
& \leq \frac{c}{2 \delta}\left\|P^{(1)}-P^{(2)}\right\|_{L^{2}(0, T)}^{2}+\frac{\delta}{2} \int_{0}^{t}\left(\left\|w_{x t}\right\|^{2}+\left\|w_{t}\right\|^{2}\right)
\end{aligned}
$$

By adjusting $\delta>0$ small enough (in dependence solely on $R$ ), we obtain directly from (12)-(13), that for all $t \in(0, T)$

$$
\begin{aligned}
\left\|w_{x}(t)\right\|^{2}+\left\|w_{t}(t)\right\|^{2}+ & \int_{0}^{t}\left\|w_{x t}(s)\right\|^{2} d s \\
\leq & c\left(\left\|P^{(1)}-P^{(2)}\right\|_{L^{2}(0, T)}^{2}+\left\|f^{(1)}-f^{(2)}\right\|_{L^{2}\left(\Omega_{T}\right)}^{2}\right)+ \\
& \quad+c \int_{0}^{t}\left(\|\xi(s)\|^{2}+\left\|\xi_{x}(s)\right\|^{2}+\left\|w_{t}(s)\right\|^{2}+\left\|w_{x}(s)\right\|^{2}\right) d s .
\end{aligned}
$$

Next we substitute $\theta=\xi$ into (10) and integrate over $(0, T)$, we get

$$
\begin{aligned}
\frac{1}{2}\|\xi(t)\|^{2}+ & D \int_{0}^{t}\left\|\xi_{x}(s)\right\|^{2}+\int_{0}^{t} \int_{\Omega}\left(\mu_{2}\left(\chi^{(1)}\right)-\mu_{2}\left(\chi^{(2)}\right)\right) \xi d x d s+k \int_{0}^{t} \int_{\Gamma} \xi^{2} d x \\
& =\int_{0}^{t} \int_{\Omega}\left(\phi\left(u_{x}^{(1)}\right) \xi+\left(K-\chi^{(2)}\right)\left(\phi\left(u_{x}^{(1)}\right)-\phi\left(u_{x}^{(2)}\right)\right)\right) \xi d x d s+k \int_{0}^{t} \int_{\Gamma}\left(\chi_{\Gamma}^{(1)}-\chi_{\Gamma}^{(2)}\right) \xi d x
\end{aligned}
$$

By the same calculation as above, the right-hand side is bounded by:

$$
c\left(\int_{0}^{t}\left(\|\xi(s)\|^{2}+\left\|w_{x}(s)\right\|^{2}\right)+\left\|\chi_{\Gamma}^{(1)}-\chi_{\Gamma}^{(2)}\right\|_{\left(L^{2}(0, T)\right)^{2}}^{2}\right) .
$$

Finally we test (10) with $\theta=\xi_{t}$. After integration over $(0, T)$, we obtain

$$
\begin{aligned}
\left\|\xi_{x}(t)\right\|^{2}+\int_{\Gamma} \xi^{2} d x+ & \int_{0}^{t}\left\|\xi_{t}(s)\right\|^{2} \\
& \leq c \int_{0}^{t}\left(\|\xi(s)\|^{2}+\left\|w_{x}(s)\right\|^{2}+\left\|\chi_{\Gamma}^{(1)}-\chi_{\Gamma}^{(2)}\right\|_{\left(H^{1}(0, T)\right)^{2}}^{2}\right) .
\end{aligned}
$$

Adding (14), (15) and (16), shows the existence of some constant $c>0$ such that there holds for any $t \in(0, T)$

$$
\begin{aligned}
\left\|w_{x}(t)\right\|^{2} & +\left\|w_{t}(t)\right\|^{2}+\|\xi(t)\|^{2}+\left\|\xi_{x}(t)\right\|^{2}+\|\xi(t)\|_{L^{2}(\Gamma)}^{2}+ \\
& +\int_{0}^{t}\left(\left\|\xi_{x}(s)\right\|^{2}+\left\|\xi_{t}(s)\right\|^{2}+\|\xi(s)\|_{L^{2}(\Gamma)}^{2}+\left\|w_{x t}(s)\right\|^{2}\right) d s \\
& \leq c\left(\left\|P^{(1)}-P^{(2)}\right\|_{L^{2}(0, T)}^{2}+\left\|f^{(1)}-f^{(2)}\right\|_{L^{2}\left(\Omega_{T}\right)}^{2}+\left\|\chi_{\Gamma}^{(1)}-\chi_{\Gamma}^{(2)}\right\|_{\left(H^{1}(0, T)\right)^{2}}^{2}+\right. \\
& \left.\quad+\int_{0}^{t}\left(\|\xi(s)\|^{2}+\left\|\xi_{x}(s)\right\|^{2}+\left\|w_{x}(s)\right\|^{2}+\left\|w_{t}(s)\right\|^{2}\right) d s\right)
\end{aligned}
$$

Hence by Gronwall's inequality, we can conclude the estimate of Theorem 3.1 . 


\subsection{Optimal Control}

Let $T>0$ be given and suppose $(\mathrm{H} 1)-(\mathrm{H} 10)$ are satisfied. Then, by Theorem 2.1, to any $\left(f, P, \chi_{\Gamma}\right)$ in $L^{2}\left(\Omega_{T}\right) \times L^{2}(0, T) \times\left(H^{1}(0, T)\right)^{2}$, the system (1)-(6) admits a unique global weak solution $(u, \chi)$ on $\Omega_{T}=\Omega \times(0, T)$.

Our aim is to formulate an optimal control for the system (1)-(6) using the data $f, P$ and $\chi$ as control variables. Indeed, the observations all suggest a connection between mechanical, electrical (neglected in our case) and ionic variables (primarily calcium and protons) in the control of cellular growth and morphogenesis. Therefore, it is natural to consider the following cost functional involving the order parameters $u, \varepsilon=u_{x}$ and $\chi$, as well as the natural control variables $f, P$ and $\chi_{\Gamma}$ :

$$
\begin{aligned}
\boldsymbol{J}\left(f, P, \chi_{\Gamma}\right)= & \alpha_{1} \iint_{\Omega_{T}}\left|\chi-\bar{\chi}_{1}\right|^{2} d x d t+\alpha_{2} \int_{0}^{T} \int_{\Gamma}\left|\chi-\bar{\chi}_{1}\right|^{2} d x d t \\
& \left.+\alpha_{3} \int_{\Omega} \mid \chi(x, T)-\bar{\chi}_{3}(x)\right)\left.\right|^{2} d x d t+\alpha_{4} \iint_{\Omega_{T}}\left|u_{t}-\bar{u}_{1}\right|^{2} d x d t \\
& \left.+\alpha_{5} \iint_{\Omega_{T}}\left|u_{x}-\bar{u}_{2}\right|^{2} d x d t+\alpha_{6} \int_{\Omega} \mid u_{x}(x, T)-\bar{u}_{3}(x)\right)\left.\right|^{2} d x d t \\
& +\alpha_{7}\|f\|_{L^{2}\left(\Omega_{T}\right)}+\alpha_{8}\|P\|_{L^{2}(0, T)}+\alpha_{9}\left\|\chi_{\Gamma}\right\|_{\left(H^{1}(0, T)\right)^{2}} .
\end{aligned}
$$

The control problem consists in finding $\left(\bar{f}, \bar{P}, \bar{\chi}_{\Gamma}\right) \in L^{2}\left(\Omega_{T}\right) \times L^{2}(0, T) \times\left(H^{1}(0, T)\right)^{2}$ that minimizes the cost functional $\boldsymbol{J}\left(f, P, \chi_{\Gamma}\right)$ subject to the constraint that $(u, \chi)$ is the weak solution of (1)-(6), corresponding to $\left(f, P, \chi_{\Gamma}\right) . \alpha_{i} \geq 0, i=1 \ldots 6$ and $\alpha_{7}, \alpha_{8}, \alpha_{9}>0$ are given constants.

Theorem 3.2. Let the assumptions of Theorem 2.1 be satisfied. Then $\mathbf{J}$ has a minimun in $L^{2}\left(\Omega_{T}\right) \times L^{2}(0, T) \times\left(H^{1}(0, T)\right)^{2}$.

Proof. Let $\left(f^{m}, P^{m}, \chi_{\Gamma}^{m}\right)$ be a minimizing sequence. Then, there exists a subsequence, still denoted by $\left(f^{m}, P^{m}, \chi_{\Gamma}^{m}\right)$ such that:

$$
\begin{aligned}
& f^{m} \longrightarrow \tilde{f} \text { in } L^{2}\left(0, T, L^{2}(\Omega)\right) \text { weak, } \\
& P^{m} \longrightarrow \widetilde{P} \text { in } L^{2}(0, T) \text { weak. } \\
& \chi_{\Gamma}^{m} \longrightarrow \tilde{\chi}_{\Gamma} \text { in }\left(H^{1}(0, T)\right)^{2} \text { weak }
\end{aligned}
$$

The corresponding solution $\left(u^{m}, \chi^{m}\right)$ of $\left(f^{m}, P^{m}, \chi_{\Gamma}^{m}\right)$ satisfy the estimations (cf [6]):

$$
\begin{array}{llll}
\left\|u_{t t}^{m}\right\|_{L^{2}\left(0, T, L^{2}(\Omega)\right)} \leq c & ; & & \left\|\chi_{t}^{m}\right\|_{L^{2}\left(0, T, L^{2}(\Omega)\right)} \leq c, \\
\left\|u^{m}\right\|_{L^{2}\left(0, T, L^{2}(\Omega)\right)} \leq c & ; & \left\|\chi_{x}^{m}\right\|_{L^{2}\left(0, T, L^{2}(\Omega)\right)} \leq c, \\
\left\|u_{x t}^{m}\right\|_{L^{2}\left(0, T, L^{2}(\Omega)\right)} \leq c & ; & \left\|\chi_{\mid \Gamma}^{m}\right\|_{L^{2}\left(0, T, L^{2}(\Gamma)\right)} \leq c,
\end{array}
$$

and

$$
\begin{array}{lll}
\left\|u_{x}^{m}\right\|_{L^{\infty}\left(0, T, L^{2}(\Omega)\right)} \leq c & ; & \left\|\chi_{x}^{m}\right\|_{L^{\infty}\left(0, T, L^{2}(\Omega)\right)} \leq c \\
\left\|u_{x x}^{m}\right\|_{L^{\infty}\left(0, T, L^{2}(\Omega)\right)} \leq c & ; & \left\|\chi_{\mid \Gamma}^{m}\right\|_{L^{\infty}\left(0, T, L^{2}(\Gamma)\right)} \leq c \\
\left\|u_{x t}^{m}\right\|_{L^{2}\left(0, T, L^{\infty}(\Omega)\right)} \leq c & ; & \left\|\chi_{t}^{m}\right\|_{L^{2}\left(0, T, L^{\infty}(\Omega)\right)} \leq c
\end{array}
$$


where $\chi_{\mid \Gamma}^{m}$ is the restriction of $\chi^{m}$ to $\Gamma$.

By using Poincaré's inequality, we can conclude

$$
\left\|\chi^{m}\right\|_{L^{2}(\Omega)} \leq c\left(\left\|\chi_{x}^{m}\right\|_{L^{2}(\Omega)}^{2}+\left\|\chi_{\mid \Gamma}^{m}\right\|_{L^{2}(\Gamma)}^{2}\right)^{\frac{1}{2}}
$$

This estimates lead with the help of the Rellich-Kondrachov Theorem (since $\Omega$ is one dimensional) to the bounds:

$$
\left\|\chi^{m}\right\|_{\mathcal{C}\left(\bar{\Omega}_{T}\right)} \leq c \sup _{t \in[0, T]}\left(\left\|\chi_{x}^{m}\right\|_{L^{2}(\Omega)}^{2}+\left\|\chi_{\mid \Gamma}^{m}\right\|_{L^{2}(\Gamma)}^{2}\right)^{\frac{1}{2}} \leq c \quad \text { and } \quad\left\|u_{x}^{m}\right\|_{\mathcal{C}\left(\bar{\Omega}_{T}\right)} \leq c
$$

where $c$ is positive constant.

It follows from the estimates (17) that $\left\{\left(u^{m}, \chi^{m}\right)\right\}_{m}$ contain a subsequence, still denoted by $\left\{\left(u^{m}, \chi^{m}\right)\right\}_{m}$, such that

as well as

$$
\begin{aligned}
& u_{t t}^{m} \longrightarrow \widetilde{u}_{t t} \text { in } L^{2}\left(0, T, L^{2}(\Omega)\right) \text { weak, } \\
& u_{x t}^{m} \longrightarrow \widetilde{u}_{x t} \text { in } L^{2}\left(0, T, L^{2}(\Omega)\right) \text { weak, } \\
& u^{m} \longrightarrow \widetilde{u} \text { in } L^{2}\left(0, T, L^{2}(\Omega)\right) \text { weak, }
\end{aligned}
$$

$$
\begin{aligned}
& \chi_{t}^{m} \longrightarrow \widetilde{\chi}_{t} \text { in } L^{2}\left(0, T, L^{2}(\Omega)\right) \text { weak, } \\
& \chi_{x}^{m} \longrightarrow \widetilde{\chi}_{x} \text { in } L^{2}\left(0, T, L^{2}(\Omega)\right) \text { weak, } \\
& \chi_{\mid \Gamma}^{m} \longrightarrow \widetilde{\chi}_{\mid \Gamma} \text { in } L^{2}\left(0, T, L^{2}(\Gamma)\right) \text { weak. }
\end{aligned}
$$

Indeed, because of the estimate (19), $\left\{u_{x}^{m}\right\}_{m \geq 1}$ is a bounded subset of $\mathcal{C}\left(\bar{\Omega}_{T}\right)$. Thanks to the Ascoli Theorem, it remains to show that $\left\{u_{x}^{m}\right\}_{m \geq 1}$ is a uniform equicontinuous subset.

From (18), and the Hölder inequality, we deduce that

$$
\begin{aligned}
\left|u_{x}^{m}\left(x_{1}, t_{1}\right)-u_{x}^{m}\left(x_{2}, t_{2}\right)\right| & \leq \int_{t_{1}}^{t_{2}}\left|u_{x t}^{m}\left(x_{1}, s\right)\right| d s+\int_{x_{1}}^{x_{2}}\left|u_{x x}^{m}\left(r, t_{2}\right)\right| d r \\
& \leq \sqrt{\left|t_{2}-t_{1}\right|}\left\|u_{x t}^{m}\left(x_{1}, .\right)\right\|_{L^{2}(0, T)}+\sqrt{\left|x_{2}-x_{1}\right|}\left\|u_{x x}^{m}\left(., t_{2}\right)\right\|_{L^{2}(\Omega)} \\
& \leq c\left(\sqrt{\left|t_{2}-t_{1}\right|}+\sqrt{\left|x_{2}-x_{1}\right|}\right) \quad \forall\left(\left(x_{1}, t_{1}\right) ;\left(x_{2}, t_{2}\right)\right) \in \bar{\Omega}_{T}^{2} .
\end{aligned}
$$

where $c$ is independent of $m, x_{1}, x_{2}, t_{1}$, and $t_{2}$.

Arguing in a similar way, it is not difficult to check that

$$
\left|\chi^{m}\left(x_{1}, t_{1}\right)-\chi^{m}\left(x_{2}, t_{2}\right)\right| \leq c\left(\sqrt{\left|t_{2}-t_{1}\right|}+\sqrt{\left|x_{2}-x_{1}\right|}\right) .
$$

Hence by (H1) and (H3), we have that

$$
\left.\begin{array}{l}
\frac{\partial \Psi}{\partial \varepsilon}\left(u_{x}^{m}, \chi^{m}\right) \longrightarrow \frac{\partial \Psi}{\partial \varepsilon}\left(\widetilde{u}_{x}, \widetilde{\chi}\right) \\
\mu_{1}\left(\chi^{m}\right) \longrightarrow \mu_{1}(\widetilde{\chi}) \\
E\left(\chi^{m}\right) \longrightarrow E(\widetilde{\chi}) \\
\phi\left(u_{x}^{m}\right) \longrightarrow \phi\left(\widetilde{u}_{x}\right) \\
\mu_{2}\left(\chi^{m}\right) \longrightarrow \mu_{2}(\widetilde{\chi})
\end{array}\right\} \text { uniformly on } \bar{\Omega}_{T}
$$

Hence its follows

$$
\left.\begin{array}{l}
\mu_{1}\left(\chi^{m}\right) u_{x t}^{m} \longrightarrow \mu_{1}(\widetilde{\chi}) \widetilde{u}_{x t} \\
E\left(\chi^{m}\right) u^{m} \longrightarrow E(\widetilde{\chi}) \widetilde{u}
\end{array}\right\} \text { weakly in } L^{2}\left(\Omega_{T}\right)
$$

By passing to the limit as $m \longrightarrow+\infty$ in (7)-(8), we obtain that $(\widetilde{u}, \widetilde{\chi})$ is the weak solution of (1)-(6) corresponding to $\left(\widetilde{f}, \widetilde{P}, \tilde{\chi}_{\Gamma}\right)$. 
To conclude the proof, observe that the functional $\left(\alpha_{7}\|f\|_{L^{2}\left(\Omega_{T}\right)}^{2}+\alpha_{8}\|P\|_{L^{2}(0, T)}^{2}+\alpha_{9}\left\|\chi_{\Gamma}\right\|_{\left(H^{1}(0, T)\right)^{2}}^{2}\right)^{\frac{1}{2}}$ defines the norm on the Hilbert space $L^{2}\left(\Omega_{T}\right) \times L^{2}(0, T) \times\left(H^{1}(0, T)\right)^{2}$ and is thus weakly lower semicontinuous. Consequently $\boldsymbol{J}\left(\widetilde{f}, \widetilde{P}, \widetilde{\chi}_{\Gamma}\right) \leq \liminf _{m \rightarrow+\infty} \boldsymbol{J}\left(f^{m}, P^{m}, \chi^{m}\right)$, and the assertion is proved.

\section{REFERENCES}

[1] C.Brière, Dynamics of the Goodwin-Trainor mechanochemical model, Acta Biotheoret. 42 (1994), 137-146

[2] C.Brière and B.C.Goodwin, Geometry and dynamics of tip morphogenesis in acetabularia, J.Theoret. Biol. 131 (1988), 461475 .

[3] C.Brière et B.C.Goodwin, Effects of calcium input/output on the stability of a system for calcium regulated viscoelastic strain fields, J. Math. Biol. 28 (1990), 585-593.

[4] P.Colli, Global existence results for a mathematical model of cell morphogenesis in calcium-regulated strain fields, J.Math. Anal. Appl. Sci. 190, (1995), 220-243.

[5] R.Dautray and J.L.Lions, Analyse mathématique et calcul numérique pour les sciences et les techniques, Vol. 3, Masson, Paris, 1985.

[6] L.Ghannam, Résultat d'existence globale et d'unicité pour un modèle de morphogenèse cellulaire, $V^{a s}$ Jornadas Zaragoza-Pau de Matemática Aplicada y Estadística, Jaca (1997), 303-310

[7] B.C.Goodwin and L.E.H.Trainor, Typ and whorl morphogenesis in Acetabularia, J. Theoret. Biol. 117 (1985), 79-106.

[8] B.C.Goodwin and L.E.H.Trainor, Stability analysis of a set of calcium-regulated viscoelastic equations, Physica, D21 (1986), $137-145$.

[9] T.Hart, L.E.H.Trainor, and B.C.Goodwin, Diffusion effects in calcium-regulated strain fields, J. Theoret. Biol. 136 (1989), 327-336.

[10] J.L.Lions, Quelques méthodes de résolution des problèmes aux limites non linéaires, Dunod Gauthier-Villars,Paris, 1969.

[11] M.Niezgodka and J.Sprekels, Existence of solutions for a mathematical model of structural phase transitions in shape memory alloys, Math. Meth. Appl. Sci.Vol .10 (1988), 197-223 .

[12] G.F.Oster and G.M.Odell, The mechanochemistry of cytogels, Phys. D 12 (1984), 333-350. 